\title{
Tumor-microenvironment induced all-in-one nanoplatform for multimodal imaging-guided chemical and photothermal therapy of cancer
}

Xiaodong Lin, ${ }^{\dagger}$ Yuan Fang, ${ }^{\dagger}$ Zhanhui Tao, ${ }^{\dagger}$ Xia Gao, ${ }^{\dagger}$ Tianlin Wang, ${ }^{\dagger}$ Minyang Zhao, ${ }^{\dagger}$ Shuo Wang, * $*$ and Yaqing Liu* $* \$$

†State Key Laboratory of Food Nutrition and Safety, College of Food Engineering and Biotechnology, Tianjin University of Science and Technology, Tianjin 300457, P. R. China.

Tianjin Key Laboratory of Food Science and Health, School of Medicine, Nankai University, Tianjin, 300071, P. R. China.

§Beijing Advanced Innovation Center for Food Nutrition and Human Health, Beijing Technology and Business University, Beijing 100037, P. R. China.

*Corresponding authors: E-mail: yaqingliu@tust.edu.cn (Yaqing Liu), wangshuo@nankai.edu.cn (Shuo Wang) 
Materials. Cupric nitrate $\left[\mathrm{Cu}\left(\mathrm{NO}_{3}\right)_{2}\right], \mathrm{GSH}$, and PEG (molecular weight: 2000) were purchased from Sinopharm Chemical Reagent Co.,Ltd., Sodium sulfide $\left(\mathrm{Na}_{2} \mathrm{~S}\right)$ was obtained from Aladdin Bio-chem technology Co.,Ltd.. Poly vinylpyrrolidone (PVP K30), doxorubicin (DOX), Calcein-AM/PI test, and 3-(4,5-dimethylthiazol-2-yl)-2,5-diphenyltetrazolium bromide (MTT) were obtained from Beijing Solarbio Technology Co.,Ltd.. Fetal Bovine Serum (FBS) and Dulbecco's modified Eagle's medium (DMEM) were provided by Gibco Invitrogen Corp. Hydrazine Monohydrate was purchased from TCI (Shanghai) Development Co.,Ltd.. All the chemicals were used without further purification.

Instruments : Ultraviolet-visible-NIR (UV-vis-NIR) spectra were recorded by a Cary 50-Bio UV spectrometer (Victoria, Australia). Transmission electron microscopy (TEM) images were obtained with a 2010FEF microscope (JEOL, Japan). X-ray photoelectron spectroscopic (XPS) measurements were performed on PHI-5000 Versaprobe (PHI, Japan). Fluorescence spectra were recorded with a LUMINA Fluorescence Spectrometer (Thermo, USA). MR images were obtained by Siemens Prisma 3.0 T MR scanner (Erlangen, Germany). The fluorescence biodistribution of DOX in mice were monitored by in vivo imaging system (Berthold LB 983, Germany). Fluorescence images of live/dead co-staining experiment were performed with the Fluorescence confocal microscope (Nikon). 

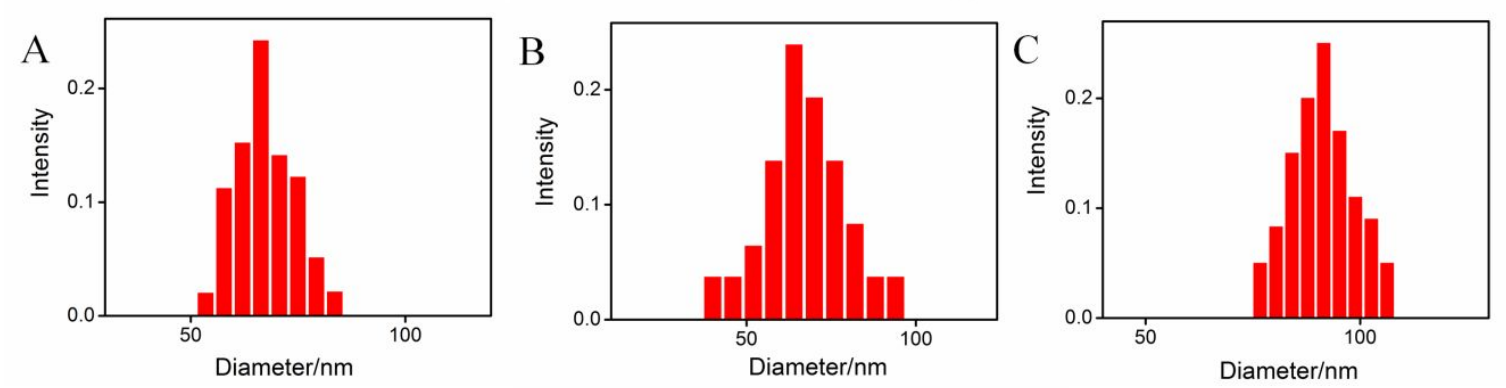

Figure S1. The DLS (Dynamic Light Scattering) of $\mathrm{CuS}_{\mathrm{NC}}$ (A), $\mathrm{CuS}_{\mathrm{NC}} @ \mathrm{DOX}$ (B) and $\mathrm{CuS}_{\mathrm{NC}} @ \mathrm{DOX} @ \mathrm{MnO}_{2-\mathrm{NS}}(\mathrm{C})$.

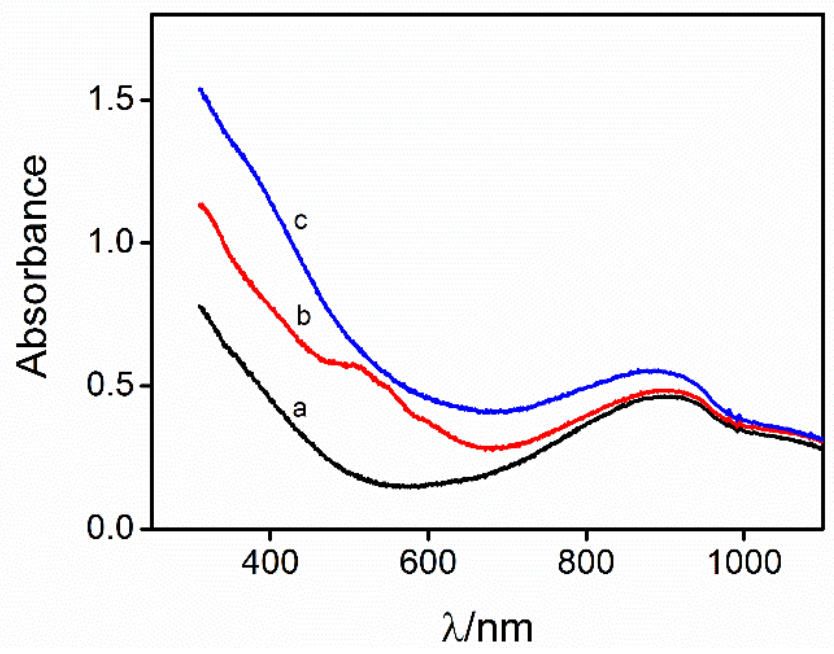

Figure S2. UV-vis absorption spectra of $\mathrm{CuS}_{\mathrm{NC}}(\mathrm{a}), \mathrm{CuS}_{\mathrm{NC}} @ \mathrm{DOX}$ (b) and $\mathrm{CuS}_{\mathrm{NC}} @ \mathrm{DOX} @ \mathrm{MnO}_{2-\mathrm{NS}}(\mathrm{c})$. 

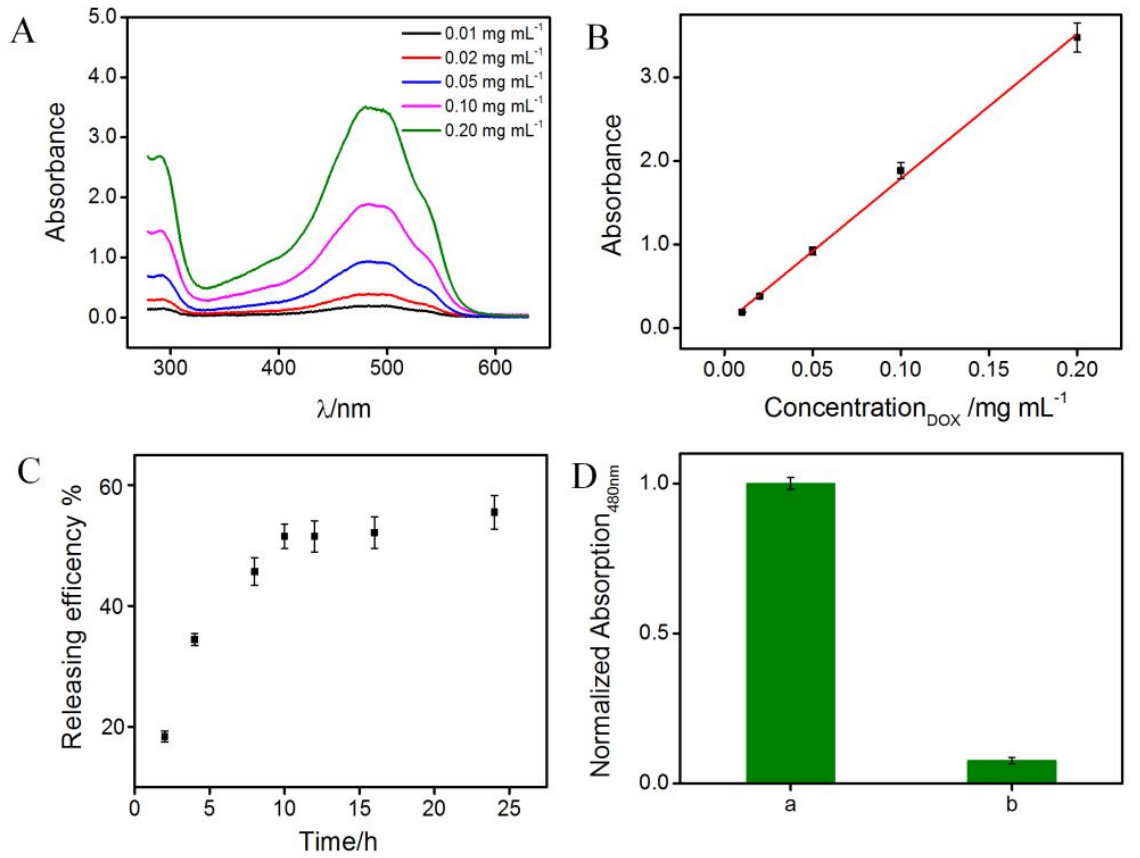

Figure S3. (A) The UV-vis absorption spectra of DOX with different concentrations. (B) The absorption at $480 \mathrm{~nm}$ against the concentration of DOX. (C) The releasing efficiency of DOX from $\mathrm{CuS}_{\mathrm{NC}}$ against incubation time. (D) The normalized absorption at $480 \mathrm{~nm}$ of the loaded DOX in the nanoplatform (a), and supernatant solution of DOX after incubating the nanoplatform in serum for three days (b).
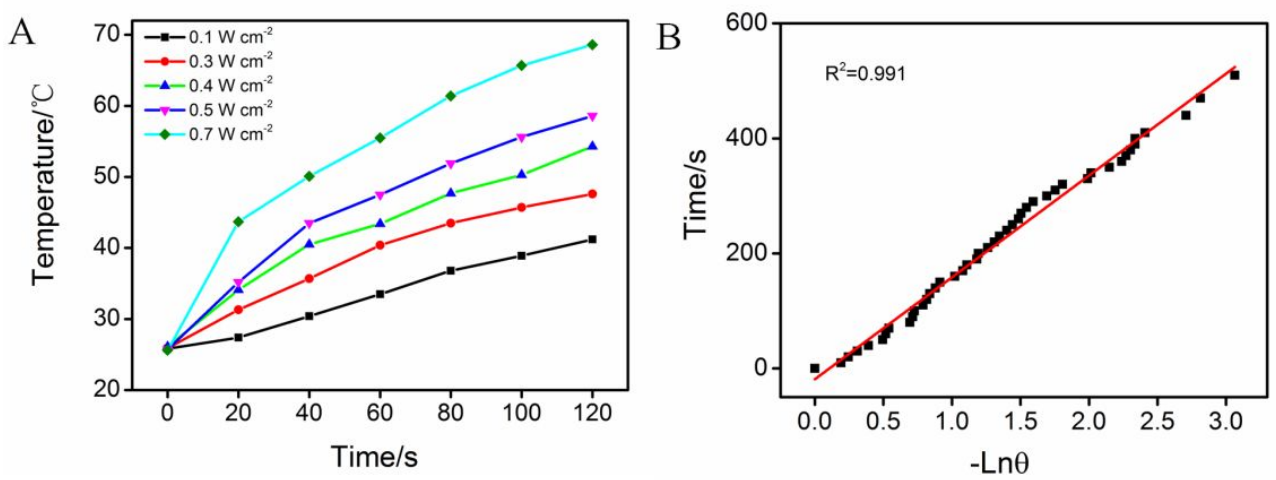

Figure S4. (A) Temperature of $\mathrm{CuS}_{\mathrm{NC}} @ \mathrm{DOX} @ \mathrm{MnO}_{2-\mathrm{NS}}$ aqueous solution $\left(200 \mu \mathrm{g} \mathrm{m}^{-1}\right)$ under irradiation of NRI with various power density. (B) Linear time data versus $-\ln \theta$ obtained from the cooling period. 

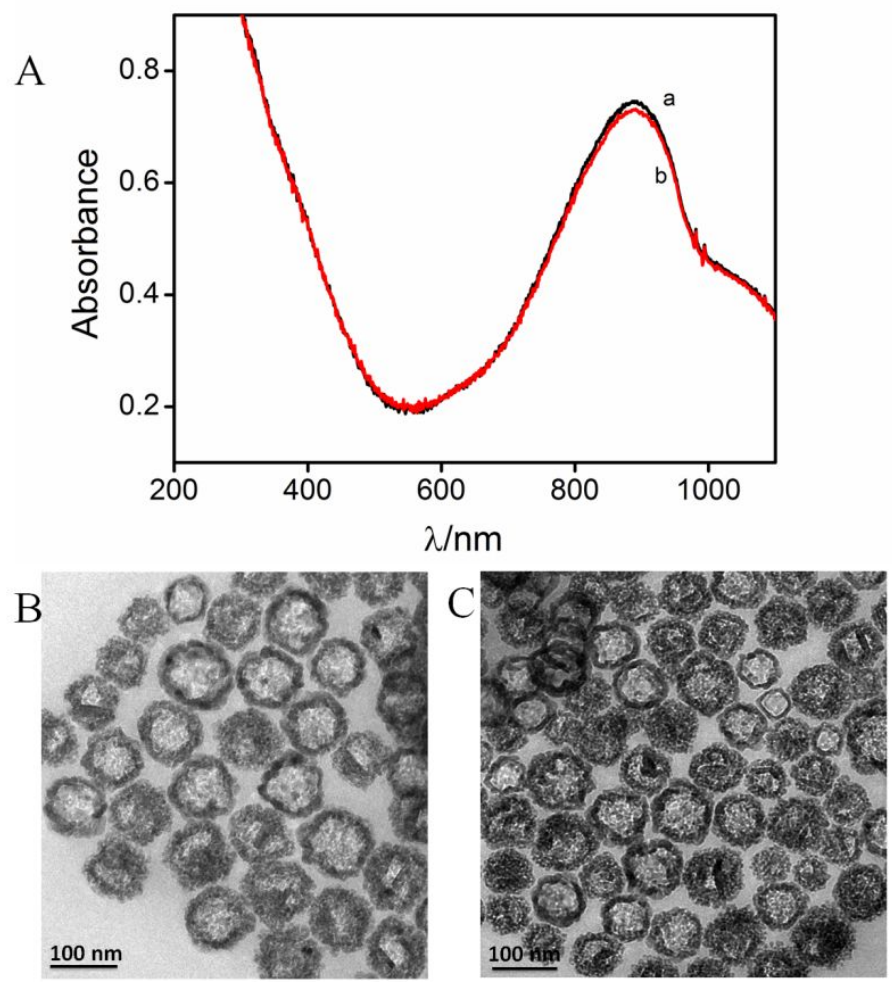

Figure S5. (A) UV-vis-NIR absorption spectra of $\mathrm{CuS}_{\mathrm{NC}}$ before (a) and after (b) being NIR irradiated (808 $\mathrm{nm}$ ) for $15 \mathrm{~min}$. The corresponding TEM imaging of $\mathrm{CuS}_{\mathrm{NC}}$ before (B) and after (C) being NIR irradiated $(808 \mathrm{~nm})$ for $15 \mathrm{~min}$.

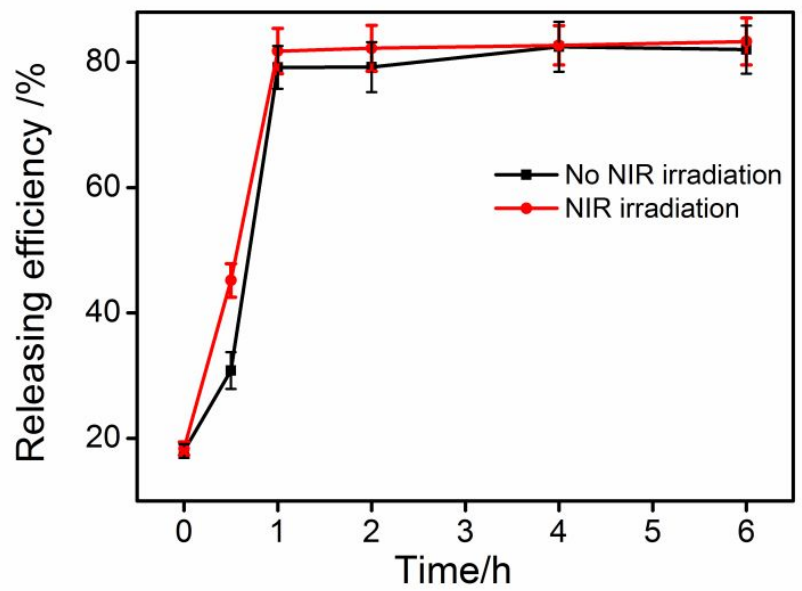


Figure S6. The DOX releasing efficiency of $\mathrm{CuS}_{\mathrm{NC}} @ \mathrm{DOX} @ \mathrm{MnO}_{2-\mathrm{NS}}$ in PBS (pH 5.5) containing GSH before (black curve) and after NIR laser treatment (red curve).

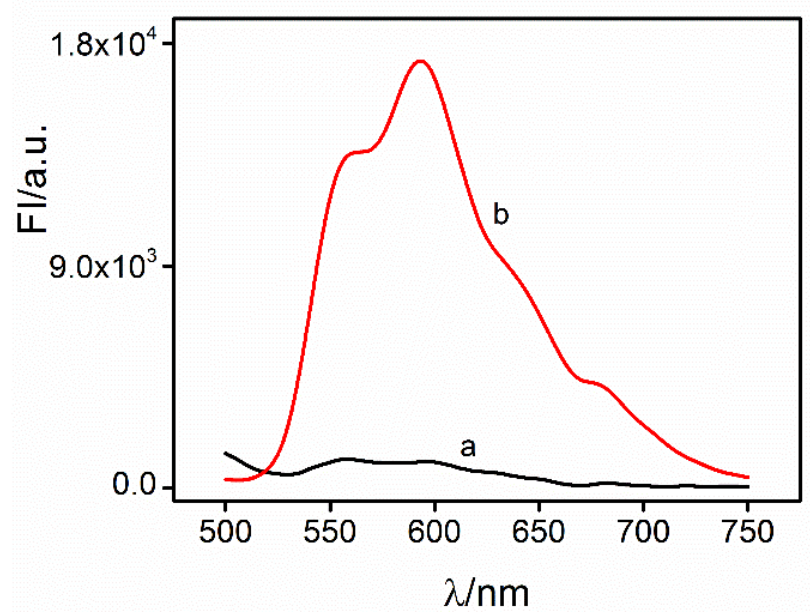

Figure S7. The fluorescence spectra of $\mathrm{CuS}_{\mathrm{NC}} @ \mathrm{DOX} @ \mathrm{MnO}_{2-\mathrm{NS}}$ in absence (a) and presence (b) of GSH $(5.0 \mathrm{mM})$

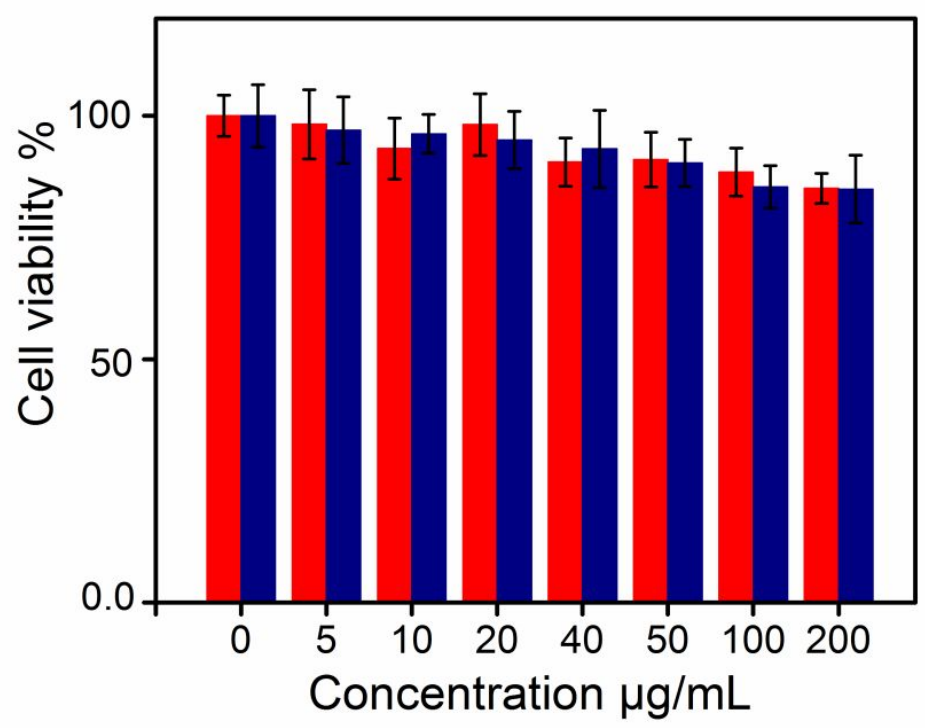

Figure S8. MTT assay evaluating cytotoxicity of $\mathrm{CuS}_{\mathrm{NC}}$ (red column) and $\mathrm{CuS}_{\mathrm{NC}} @ \mathrm{DOX} @ \mathrm{MnO}_{2-\mathrm{Ns}}$ (blue 
column) at different concentrations by incubating with L-02 cells for $24 \mathrm{~h}$.

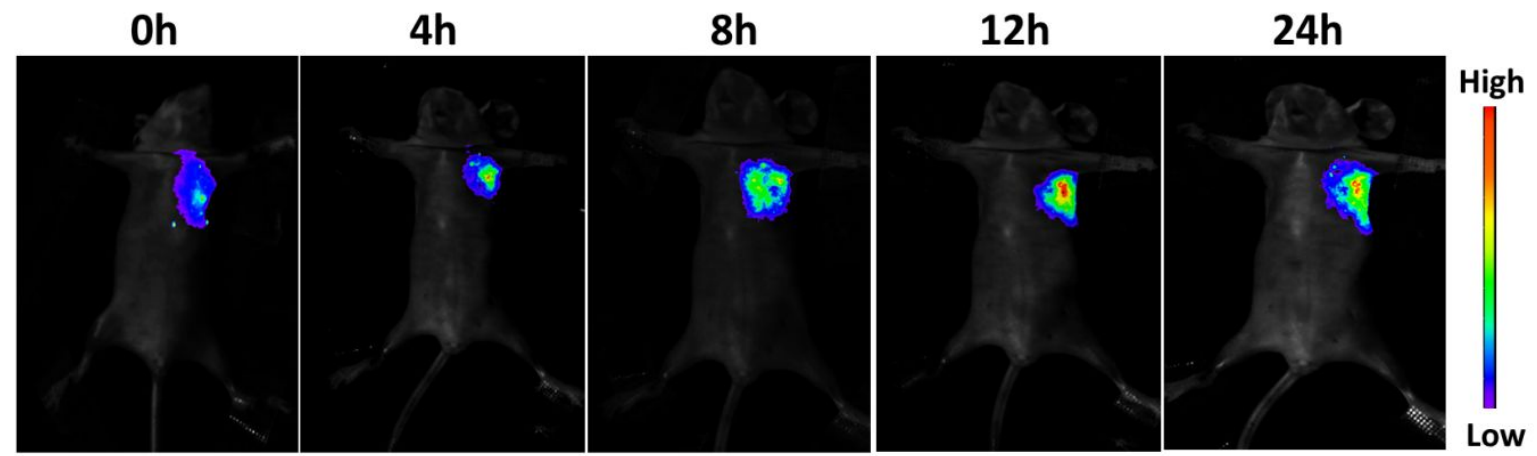

Figure S9. The in vivo fluorescence image of mice with intravenous injection the $\mathrm{CuS}_{\mathrm{NC}} @ \mathrm{DOX} @ \mathrm{MnO}_{2-\mathrm{NS}}$ from 0 to $24 \mathrm{~h}$. 